Provided for non-commercial research and education use. Not for reproduction, distribution or commercial use.

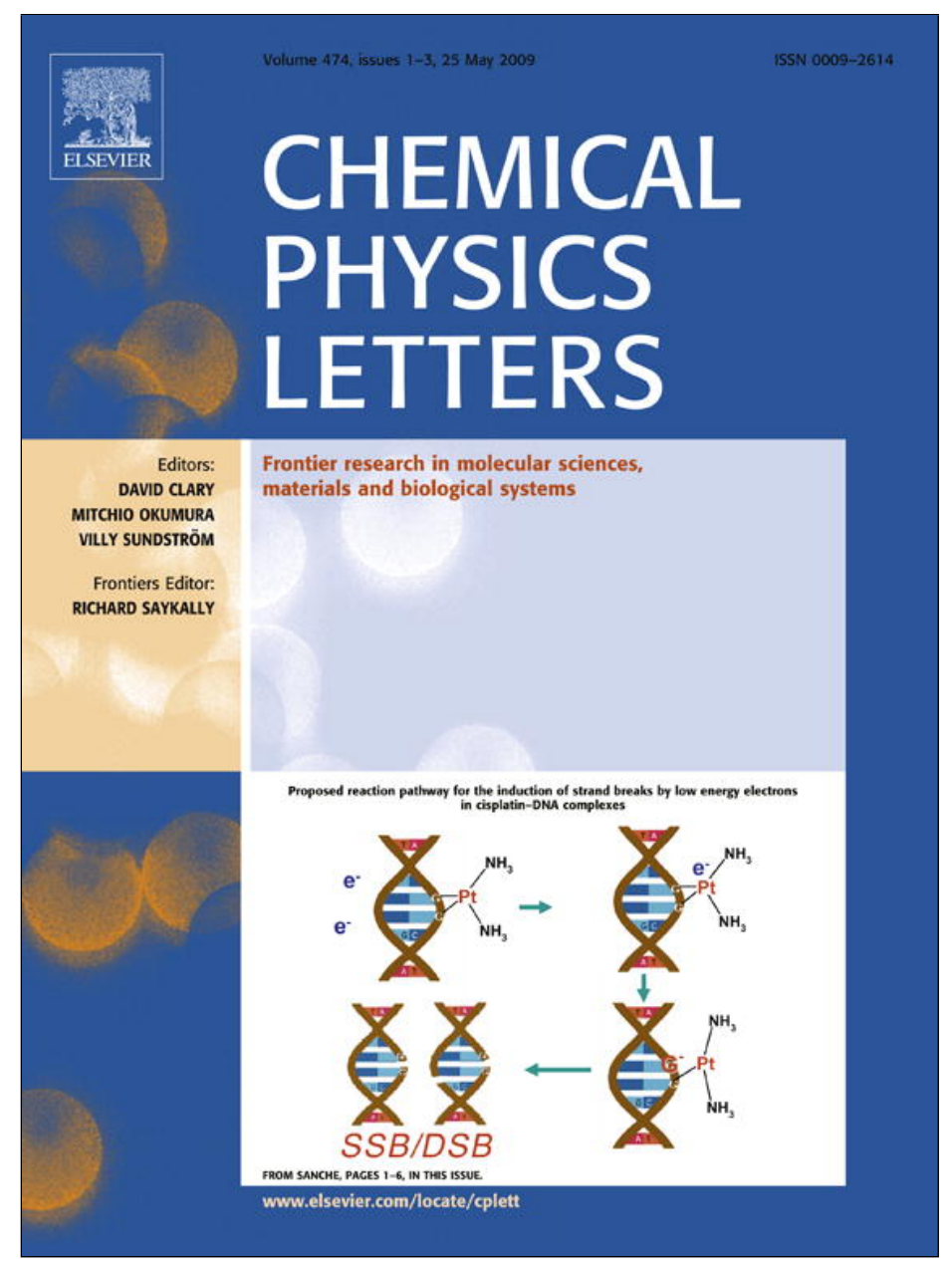

This article appeared in a journal published by Elsevier. The attached copy is furnished to the author for internal non-commercial research and education use, including for instruction at the authors institution and sharing with colleagues.

Other uses, including reproduction and distribution, or selling or licensing copies, or posting to personal, institutional or third party websites are prohibited.

In most cases authors are permitted to post their version of the article (e.g. in Word or Tex form) to their personal website or institutional repository. Authors requiring further information regarding Elsevier's archiving and manuscript policies are encouraged to visit:

http://www.elsevier.com/copyright 


\title{
Observation of ultrafast internal conversion in fullerene anions in solution
}

\author{
Michael J. Hope ${ }^{a}$, Michael P. Higlett ${ }^{a}$, David L. Andrews ${ }^{a}$, Stephen R. Meech ${ }^{a, *}$, Ian D. Hands ${ }^{b}$, \\ Janette L. Dunn ${ }^{\mathrm{b}}$, Colin A. Bates ${ }^{\mathrm{b}}$ \\ ${ }^{a}$ School of Chemical Sciences, University of East Anglia, Norwich NR4 7TJ, UK \\ ${ }^{\mathrm{b}}$ School of Physics and Astronomy, University of Nottingham, Nottingham NG7 2RD, UK
}

\section{A R T I C L E I N F O}

\section{Article history:}

Received 19 March 2009

In final form 23 April 2009

Available online 3 May 2009

\begin{abstract}
A B S T R A C T
The ultrafast decay rates of photoexcited $\mathrm{C}_{60}^{-}$and $\mathrm{C}_{60}^{2-}$ ions have been measured in the condensed phase. The mechanism for decay is internal conversion, and the decay rate is a strong function of the charge on the ion. A bottleneck in the ground state recovery has also been detected, and its interpretation is discussed.
\end{abstract}

(c) 2009 Elsevier B.V. All rights reserved.

\section{Introduction}

The spectroscopy, structure and chemistry of the negativelycharged fullerene ions $\left(C_{60}^{n-}\right.$, where $1 \leqslant n \leqslant 6$, generically referred to here as fullerides) continue to attract the attention of a large body of scientists. This is not only because of the critical role these ions play in facilitating superconductivity in certain fullerene-alkali metal salts [1], but also on account of their role in electron transfer reactions (itself a result of the high electron affinity of $C_{60}$ ) and as intermediates in fullerene photochemistry [2]. In $\mathrm{C}_{60}$, whilst a nanosecond timescale characterizes intersystem crossing from the lowest singlet state [3], relaxation amongst the higher-lying singlet states is revealed by ultrafast studies $[4,5]$. In contrast, there have been relatively few studies of the excited state dynamics of the fullerides. Fullerides can readily be generated by synthetic and electrochemical means, and in deoxygenated solutions they are relatively stable, allowing electronic spectra to be measured for solutions of anions with $n=1,2$ or 3 [6-8]. While absorption spectra have been recorded, there is only a single report of an excited state lifetime, by Ehrler et al. [9] who measured the decay of the excited monoanion in the gas phase using time-resolved two-photon two-colour photoelectron spectroscopy. In this Letter, we report measurement of the excited state decay lifetimes of the mono- and dianion of $\mathrm{C}_{60}$ in the condensed phase. To our knowledge, this is the first lifetime determination of the dianion in any medium. The ultrafast ground state recovery times that are observed allow us to draw inferences concerning both the mechanism of the radiationless decay and the strength of vibronic coupling in solution, including a dependence on the charge of the ion.

\footnotetext{
* Corresponding author. Fax: +44 1603592003.

E-mail address: s.meech@uea.ac.uk (S.R. Meech).
}

\section{Experimental}

For the majority of the measurements described here the anions were generated electrochemically. Molecular $\mathrm{C}_{60}$ (sublimed, $99.9+\%$, Aldrich) was dissolved in a 5.4:1 toluene:acetonitrile solvent containing $0.1 \mathrm{M}$ tetrabutylammonium hexafluorophosphate as a supporting electrolyte. The solution was placed in a spectroelectrochemical cell ( $2 \mathrm{~mm}$ optical pathlength) and deoxygenated by passing solvent-saturated nitrogen gas through it. It was then kept under a nitrogen atmosphere throughout the experiment. A cyclic voltammogram was recorded and reduction potentials for the mono- and dianion were determined. The results, $-0.5 \mathrm{~V}$ and $-1.0 \mathrm{~V}$, respectively, are in good agreement with the literature values $[8,10]$. Electronic absorption spectra were recorded using a near-IR-sensitive multichannel detector, setting the potential at $-0.8 \mathrm{~V}$ and $-1.3 \mathrm{~V}$ for the mono- and dianion, respectively, as shown in Fig. 1. The spectra were measured as a function of time after initiation of the reduction, while monitoring the amount of charge transferred. Once the spectra had reached a steady value, the extinction coefficient at the wavelength of maximum absorbance was determined from the Beer-Lambert law under the assumption of complete reduction to the desired anion. During the reduction of $\mathrm{C}_{60}^{-}$to $\mathrm{C}_{60}^{2-}$, an isosbestic point was observed at $990 \mathrm{~nm}$ and the $1075 \mathrm{~nm}$ peak disappeared, consistent with the presence of only two absorbing species and complete electrochemical interconversion. For $\mathrm{C}_{60}^{-}$at $1075 \mathrm{~nm}$, an extinction coefficient of $16000 \mathrm{M}^{-1} \mathrm{~cm}^{-1}$ was obtained, while for $\mathrm{C}_{60}^{2-}$ at $950 \mathrm{~nm}$, a value of $16800 \mathrm{M}^{-1} \mathrm{~cm}^{-1}$ was measured. These results, which are lower limits, are within the ranges previously reported in the literature $[8,11,12]$. From these extinction coefficients, one can predict nanosecond radiative lifetimes for both these ions.

The structure in the monoanion spectrum shown in Fig. 1a has been discussed in detail in the context of the dynamic Jahn-Teller effect in an earlier paper [13]. The spectrum comprises (at least) 4 distinct bands, assigned to the $\mathrm{T}_{1 g} \leftarrow \mathrm{T}_{1 u}$ transition in which both 


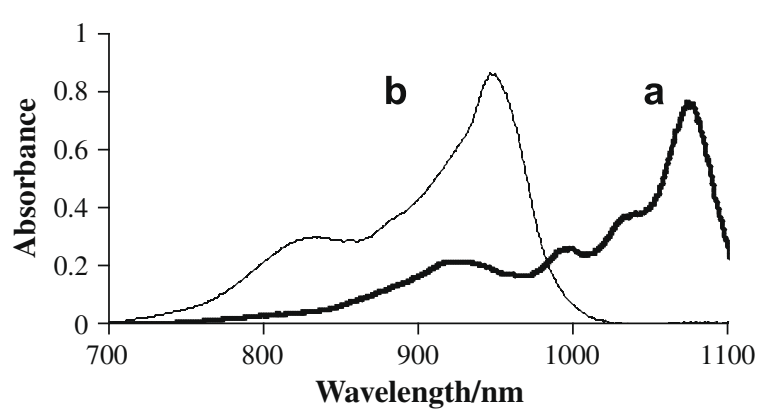

Fig. 1. Absorption spectra of electrochemically generated fullerene anions in toluene:acetonitrile solution: (a) $\mathrm{C}_{60}^{-}$(solid line), and (b) $\mathrm{C}_{60}^{2-}$ (dashed line).

ground and excited states are subject to vibronic coupling via a $\mathrm{T}_{1} \otimes \mathrm{h}$ Jahn-Teller effect. The observed spectrum was shown to be consistent with states possessing $\mathrm{D}_{3 d}$ distorted geometries.

The $\mathrm{C}_{60}^{2-}$ spectrum, Fig. $1 \mathrm{~b}$, reveals a simpler structure, with only two distinct bands being observed, both of appreciable intensity. This is consistent with a simple static Jahn-Teller coupling model proposed originally by Lawson et al. [14,15] in which the $T_{1 u}$ and $\mathrm{T}_{1 g}$ states split into $\mathrm{E}_{1 u}$ and $\mathrm{A}_{2 u}$ and $\mathrm{E}_{1 g}$ and $\mathrm{A}_{2 g}$, respectively, to yield the observed pair of transitions, $\mathrm{E}_{1 g} \leftarrow \mathrm{E}_{1 u}$ and $\mathrm{A}_{2 g} \leftarrow \mathrm{E}_{1 u}$. A more realistic dynamical treatment complementary to that attempted in Ref. [13] would be likely to yield a much more complicated structure for the bands. However, the lack of detail in the features observed precludes any attempt to produce a more realistic model.

To monitor the dynamics of the ground state recovery, we use a single-colour pump-probe experiment. The laser source was a home-built, mode-locked and cavity-dumped titanium:sapphire laser described in detail elsewhere [16]. For these measurements, intracavity mirrors were exchanged to optimize gain in the 900$1000 \mathrm{~nm}$ region. Optimum performance was at $925 \mathrm{~nm}$, producing excitation into the lowest energy excited state of the dianion, and also exciting the monoanion with only a small excess energy of approximately $1500 \mathrm{~cm}^{-1}$ (Fig. 1). At $925 \mathrm{~nm}$, the laser output consisted of ca. $40 \mathrm{fs}$ pulses of about $20 \mathrm{~nJ}$ energy per pulse at a repetition rate of $300 \mathrm{kHz}$. Pulses were split into pump and probe in the ratio $10: 1$, routed through a conventional pump-probe geometry and recombined in the spectroelectrochemical cell (spot radius ca. $100 \mu \mathrm{m}$ ). The intensity of the transmitted probe beam was monitored as a function of pump-probe delay.

\section{Results and discussion}

The transient behaviour of the monoanion solution in toluene:acetonitrile, for pump and probe wavelengths both of $925 \mathrm{~nm}$, is shown in Fig. 2a. The transmitted probe intensity increases within the 50 fs time resolution of the experiment, consistent with bleaching of the ground state. A minor, pulse-limited decrease in intensity at around time-zero is succeeded by a dominant decrease occurring on a picosecond timescale, which eventually settles to a long-lived offset that relaxes on a much longer timescale. (This slow relaxation, with a lifetime exceeding $100 \mathrm{ps,}$ could not be accurately determined, although from the observed recovery of the baseline it was established that the sample had completely relaxed before the arrival of the next pump pulse, $3 \mu$ s later.) The minor pulse-limited transient at around $t=0$ is assigned to ultrafast coherent processes in the solution (including the electronic response of the solvent). The data after $100 \mathrm{fs}$ were fitted to a single exponential relaxation plus an essentially constant offset, $I(t)=\exp (-t / \tau)+a$. The exponential recovery time, $\tau$, was determined to be $3.5 \pm 0.5$ ps with the very slowly relaxing offset $a=0.2$.
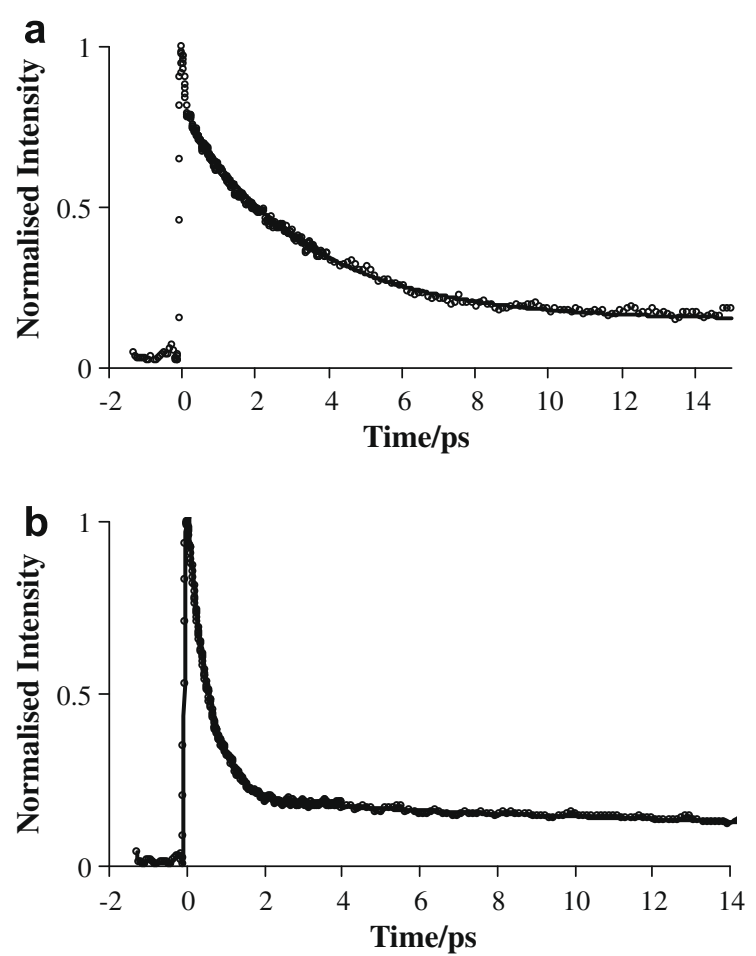

Fig. 2. Ultrafast ground state recovery of two fullerene anions: (a) the dynamics observed for $\mathrm{C}_{60}^{-}$; (b) $\mathrm{C}_{60}^{2-}$. The circles are experimental data and the solid lines fits to mono- or bi-exponential recovery kinetics (plus a constant offset on the picosecond timescale). For (a), the time-zero response has not been included in the analysis.

The pulse-limited increase in transmission followed by an exponential decrease is consistent with internal conversion (IC) from the excited state directly repopulating the ground state. These measurements on the ground state population confirm the assignment of radiationless decay through IC by Ehrler et al. [9], based on the observed ultrafast excited state decay time. The 3.5 ps ground state recovery in solution is on the same picosecond timescale as the 2.2 ps excited state decay time measured in the gas phase. This result verifies that the radiationless decay in $\mathrm{C}_{60}^{-}$is dominated by intramolecular relaxation, and that it is at most only weakly influenced by the solvent environment. Intramolecular IC may be promoted by a structural change between the ground and excited electronic states. This result thus highlights the potential significance of the Jahn-Teller distortion, since the latter provides a mechanism for generating the required difference in the excited and ground state geometries. The ground state recovery time was also measured in chemically-generated $\mathrm{C}_{60}^{-}$in THF solution and found to be $4.0 \pm 0.5 \mathrm{ps}$. This is again consistent with a negligible role for the solvent in determining the rate of IC. The implication of these results is that the key relaxation processes are intrinsic to the fulleride itself. In addition, the similarity of the excited state decay rate in the gas phase (where the $C_{60}^{-}$was excited with $3600 \mathrm{~cm}^{-1}$ excess energy), and those reported here in solution, suggests only a small effect of the excess energy on the lifetime.

Another feature also emerges in the ground state recovery cycle; the measurement in Fig. 2 reveals the development of a significant population in a comparatively long-lived intermediate state (the long-lived offset accounting for about $20 \%$ of the original bleach). Since this bottleneck state is populated on a picosecond timescale, it can be assumed to occur in a spin-allowed process, and so competition from intersystem crossing can be ruled out. There are a number of possible assignments for a bottleneck state in the ground state recovery. IC repopulates the electronic ground state with about $10^{4} \mathrm{~cm}^{-1}$ of excess energy in the accepting modes of the radiationless transition. This excess energy will initially be 
redistributed to low-frequency intramolecular modes of $\mathrm{C}_{60}^{-}$before ultimately being transferred to solvent translational and rotational motions. The vibrationally-hot state attained after intramolecular vibrational relaxation might have a different electronic spectrum to the equilibrated $\mathrm{C}_{60}^{-}$at $293 \mathrm{~K}$, leading to the observation of a long-lived change in absorbance. However, the observed offset persists for at least $100 \mathrm{ps,} \mathrm{suggesting} \mathrm{that} \mathrm{the} \mathrm{transfer} \mathrm{of} \mathrm{vibrational}$ energy from the low-frequency modes of $\mathrm{C}_{60}^{-}$to the solvent would have to be rather slow compared to the timescales observed with other large organic molecules (such as azulene [17]). This suggests an unusually weak coupling between the low-frequency modes of the solute and the solvent modes.

An alternative assignment for the bottleneck state is the population, through internal conversion, of low-lying vibronic states in the Jahn-Teller ground state manifold. In particular, a $\mathrm{D}_{3 d}$ distorted $\mathrm{C}_{60}^{-}$anion has ground vibronic states of $\mathrm{T}_{1 u}, \mathrm{~T}_{2 u}$ and $\mathrm{G}_{u}$ symmetry $[13,18]$. However, the product $T_{1 u} \otimes h_{g}$ associated with vibronic coupling between a set of electronic $\mathrm{T}_{1 u}$ states and a fivefold degenerate set of vibrations of $h_{g}$ symmetry decomposes in icosahedral symmetry as $\mathrm{T}_{1 u} \oplus \mathrm{T}_{2 u} \oplus \mathrm{G}_{u} \oplus \mathrm{H}_{u}$. Thus, there is a set of $\mathrm{H}_{u}$ levels above the ground vibronic states possessing an energy that is too high to be thermally accessible by an ion in its ground state. These vibronic states could be populated during the vibrational relaxation on the ground state surface following IC. Again, the observations require a lifetime for these states in excess of $100 \mathrm{ps}$. However, it is worth noting in this context that the ground vibronic states $\left\{\mathrm{T}_{1 u}, \mathrm{~T}_{2 u}, \mathrm{G}_{u}\right\}$ conceptually arise from combination of the ten ground state $\mathrm{D}_{3 d}$ wells that occur in the limit of strong vibronic coupling. On the other hand, the $\mathrm{H}_{u}$ states arise from nine ground state and an excited $\mathrm{D}_{3 d}$ well containing one quantum of vibration. Therefore, relaxation from the $\mathrm{H}_{u}$ states to the other states formally occurs with the loss of a quantum of vibrational energy. If the coupling to the solvent is weak, then this process may not be efficient and the $\mathrm{H}_{u}$ state relatively long-lived.

A final possibility is a photo-induced chemical reaction of the excited $\mathrm{C}_{60}^{-}$ion, leading to a change in absorbance that is irreversible on the picosecond timescale, and which recovers only through diffusion of the product out of the excited volume to be replaced by fresh, ground-state molecules. This mechanism would be consistent with the very slow recovery time. However, it is unlikely that a bimolecular process could compete with the ultrafast IC. In addition, no long-term photochemistry was detected in the steadystate absorption spectra recorded before and after irradiation. One possible mechanism for an intramolecular reaction is electron photodetachment in a multi-photon process. This is energetically feasible via a two-photon process (the electron affinity of $C_{60}$ is $2.68 \mathrm{eV}$ [19], and the photon energy is $1.34 \mathrm{eV}$ ) but since the peak power is only about $1.5 \mathrm{GW} \mathrm{cm}$ ch $^{-2}$, this mechanism requires a high photodetachment cross-section at the threshold energy. However, we did not observe a permanent change in the absorption intensity over time, as would be expected for a chemical process with a $20 \%$ yield, suggesting that any such process is reversible. Further the independence of the relaxation rate on solvent polarity is an unexpected feature if charge transfer to solvent state is significant.

In Fig. 2b, the ground state recovery for electrochemically generated $\mathrm{C}_{60}^{2-}$ is shown, again for a pump and probe wavelength of $925 \mathrm{~nm}$. The ground state recovery for the dianion is clearly much faster than for $\mathrm{C}_{60}^{-}$, the former comprising a dominant recovery time of $400 \mathrm{fs}$ followed by a minor (10\%) slower recovery of $3.0 \pm 1.5 \mathrm{ps}$ and, as was seen in the monoanion, a $20 \%$ long-lived $(>100 \mathrm{ps})$ offset. The long component can again be assigned to either a vibronically hot ground state formed during vibrational relaxation, or to the multi-photon photodetachment mechanism discussed for the monoanion.

The most remarkable feature of the photophysics of $C_{60}^{2-}$ is the very fast IC from the excited state, compared with that for $\mathrm{C}_{60}^{-}$. As already discussed by Ehrler et al. [9], the fast IC in the $\mathrm{C}_{60}^{-}$is consistent with a very strong coupling between the first excited and ground electronic state. This is in marked contrast to the case for the $C_{60}$ itself, where IC is so slow that the spin-forbidden intersystem crossing dominates the radiationless decay [4]. The present result shows that this coupling is stronger still for the dianion. This, in turn, suggests a greater Franck-Condon overlap between the excited and ground state vibronic wavefunctions, which may be a consequence of the different Jahn-Teller distortions operating in the two ions. Once again, a distortion of the nuclear framework may prove to be a key component of the mechanism for excited state decay.

In conclusion, the excited state decays of two fullerene anions have been measured in the condensed phase for the first time. The decay mechanism has been identified as ultrafast internal conversion. A comparison with the excited state lifetime of the monoanion in the gas phase [9] indicates that intramolecular relaxation dominates, and that the amount of excess energy in the initially excited state exerts only a weak influence on the lifetime. The data show that the rate of internal conversion, and by inference the vibrational coupling of ground and excited state, is sensitive to the charge on the anion. Finally, for both fullerides, a bottleneck has been detected in the ground state recovery cycle. Amongst several alternative explanations - which could not be distinguished by the present measurements - all share one striking feature; they strongly indicate that the anion photophysics is relatively insensitive to any coupling between the fulleride and solvent.

\section{Acknowledgements}

We are grateful to EPSRC for financial support for this work (Grant Nos. GR/S43894/01 and GR/S43900/01).

\section{References}

[1] O. Gunnarsson, Alkali-Doped Fullerides, Narrow-Band Solids with Unusual Properties, World Scientific, Singapore, 2004.

[2] C.A. Reed, R.D. Bolskar, Chem. Rev. 100 (2000) 1075.

[3] T.W. Ebbesen, K. Tanigaki, S. Kuroshima, Chem. Phys. Lett. 181 (1991) 501.

[4] A. Sassara, G. Zerza, M. Chergui, V. Ciulin, J.D. Ganiere, B. Deveaud, J. Chem. Phys. 111 (1999) 689.

[5] A.G. Stepanov, M.T. Portella-Oberli, A. Sassara, M. Chergui, Chem. Phys. Lett. 358 (2002) 516.

[6] G.A. Heath, J.E. McGrady, R.L. Martin, J. Chem. Soc. Chem. Commun. (1992) 1272.

[7] T. Kato et al., Chem. Phys. Lett. 186 (1991) 35.

[8] M.M. Khaled, R.T. Carlin, P.C. Trulove, G.R. Eaton, S.S. Eaton, J. Am. Chem. Soc. 116 (1994) 3465.

[9] O.T. Ehrler, J.P. Yang, C. Hättig, A. Unterreiner, H. Hippler, M. Kappes, J. Chem. Phys. 125 (2006) 074312.

[10] Q. Xie, E. Perez-Cordero, L. Echegoyen, J. Am. Chem. Soc. 114 (1992) 3978.

[11] T. Kato, Laser Chem. 14 (1993) 155.

[12] P.C. Trulove, R.T. Carlin, G.R. Eaton, S.S. Eaton, J. Am. Chem. Soc. 117 (1995) 6265.

[13] I.D. Hands, J.L. Dunn, C.A. Bates, M.J. Hope, S.R. Meech, D.L. Andrews, Phys. Rev. B 77 (2008) 115445

[14] D.R. Lawson, D.L. Feldheim, C.A. Foss, P.K. Dorhout, C.M. Elliott, C.R. Martin, B. Parkinson, J. Phys. Chem. 96 (1992) 7175.

[15] D.R. Lawson, D.L. Feldheim, C.A. Foss, P.K. Dorhout, C.M. Elliott, C.R. Martin, B. Parkinson, J. Electrochem. Soc. 139 (1992) L68.

[16] J. Garduno-Mejia, S.R. Meech, Opt. Commun. 259 (2006) 840.

[17] S.A. Kovalenko, R. Schanz, H. Hennig, N.P. Ernsting, J. Chem. Phys. 115 (2001) 3256.

[18] J.L. Dunn, C.A. Bates, Phys. Rev. B 52 (1995) 5996

[19] X.B. Wang, H.K. Woo, L.S. Wang, J. Chem. Phys. 123 (2005) 051106. 